\title{
Maximum norms of chaotic quantum eigenstates and random waves
}

\author{
R. Aurich *, A. Bäcker, R. Schubert, M. Taglieber \\ Abteilung Theoretische Physik, Universität Ulm, Albert-Einstein-Allee 11, D-89069 Ulm, Germany \\ Received 3 April 1998; received in revised form 15 June 1998; accepted 2 November 1998 \\ Communicated by C.K.R.T. Jones
}

\begin{abstract}
The growth of the maximum norms of quantum eigenstates of classically chaotic systems with increasing energy is investigated. The maximum norms provide a measure for localization effects in eigenfunctions. An upper bound for the maxima of random superpositions of random functions is derived. For the random-wave model this gives the bound $c \sqrt{\ln E}$ in the semiclassical limit $E \rightarrow \infty$. The growth of the maximum norms of random waves is compared with the growth of the maximum norms of the eigenstates of six quantum billiards which are classically chaotic. The maximum norms of these systems are numerically shown to be conform with the random-wave model. Furthermore, the distribution of the locations of the maximum norms is discussed. (1999 Elsevier Science B.V. All rights reserved.
\end{abstract}

\section{Introduction}

One of the important questions in quantum chaos is how the chaoticity of a classical system is reflected in the behavior of the eigenfunctions of the corresponding quantum system [1]. In this paper, we are concerned with bounded quantum systems on some two-dimensional Riemannian manifolds $\mathcal{M}$ governed by the stationary Schrödinger equation (with units $\hbar=2 m=1$ )

$$
-\Delta \psi_{n}(\boldsymbol{x})=E_{n} \psi_{n}(\boldsymbol{x})
$$

together with appropriate boundary conditions. Here $\Delta$ denotes the Laplace-Beltrami operator, which on the Euclidean plane is the usual Laplacian. The systems possess discrete energy spectra $\left\{E_{n}\right\}$ which we assume to be ordered by increasing value $E_{1} \leq E_{2} \leq \ldots$. In the following we assume the eigenfunctions $\psi_{n}$ to be normalized, i.e., $\left\|\psi_{n}\right\|_{2}=1$. The corresponding classical system is the geodesic flow on the tangent bundle $T \mathcal{M}$.

A model for the eigenfunctions of systems with strongly chaotic classical flow has been put forward by Berry, who conjectured that they behave like a random superposition of plane waves [2]. In the Euclidean case the random

\footnotetext{
${ }^{*}$ Corresponding author. E-mail: aurich@physik.uni-ulm.de.
} 
superposition of plane waves on a $d$-dimensional region $D \subset \mathbb{R}^{d}$ is of the form

$$
f(\boldsymbol{x})=\frac{b}{\sqrt{N}} \sum_{n=1}^{N} a_{n} \cos \left(\boldsymbol{k}_{n} \boldsymbol{x}+\varepsilon_{n}\right),
$$

where $a_{n} \in \mathbb{R}$ are assumed to be independent Gaussian random variables; $\varepsilon_{n}$ are equidistributed random variables on $\left[0,2 \pi\right.$ [, and $b$ is a real constant. The momenta $\boldsymbol{k}_{n} \in \mathbb{R}^{d}$ satisfy $\left|\boldsymbol{k}_{n}\right|=\sqrt{E}$ and are randomly equidistributed on the sphere of radius $\sqrt{E}$. For $N, E \rightarrow \infty$ the choice $b=\sqrt{2 / \operatorname{vol}(D)}$ leads to a normalized random function.

The random-wave model can be used to obtain predictions on the behavior of eigenfunctions. For example, random waves have a Gaussian amplitude distribution [2], which was found numerically for the eigenfunctions of several chaotic systems, see e.g., [3-6]. A further result which shows that almost all eigenfunctions of an ergodic system share some properties with random waves is the quantum ergodicity theorem [7-12]. It states that in classically ergodic systems almost all eigenfunctions become equidistributed in the semiclassical limit. Almost all means that the fraction of eigenfunctions which behaves differently tends to zero in the semiclassical limit. So the quantum ergodicity theorem does not exclude the possibility of localization effects in a subset of eigenfunctions, e.g., the existence of scars $[3,13]$ or bouncing-ball modes $[3,14,15]$. It is one of the most interesting open problems in the subject to determine the properties of a given classical system which are necessary to exclude any localization of eigenfunctions. If there are no exceptional subsequences of eigenfunctions this situation is called unique quantum ergodicity. This behavior is conjectured for the eigenfunctions of the Laplacian on a compact manifold of negative curvature [16,17], which is supported by numerical results [18,19].

A set of quantities which measure localization effects is given by the $L^{p}$ norms of the eigenfunctions $\psi_{n}$,

$$
\left\|\psi_{n}\right\|_{p}:=\left(\int_{\mathcal{M}} \mathrm{d} \nu\left|\psi_{n}\right|^{p}\right)^{1 / p} \text { for } p \geq 1,
$$

where $\mathrm{d} v$ is the invariant volume element on $\mathcal{M}$. Since large values of the eigenfunctions contribute stronger for larger $p$, high values of the $L^{p}$ norms for $p>2$ indicate a non-uniformly distributed eigenfunction. The maximum or $L^{\infty}$ norm $\left\|\psi_{n}\right\|_{\infty}$, which is just the maximum of the absolute value of the eigenfunction $\psi_{n}$, gives the most sensitive measure.

A general upper bound for eigenfunctions of the Laplace-Beltrami operator $\Delta$ on compact $d$-dimensional Riemannian manifolds $\mathcal{M}$ without boundary is obtained by Seeger and Sogge [20]

$$
\begin{aligned}
& \left\|\psi_{n}\right\|_{p}<c E_{n}^{\delta(p) / 2} \text { for } \frac{2(d+1)}{d-1} \leq p \leq \infty, \\
& \left\|\psi_{n}\right\|_{p}<c E_{n}^{(d-1)\left(2-p^{\prime}\right) / p^{\prime}} \quad \text { for } 2 \leq p \leq \frac{2(d+1)}{d-1},
\end{aligned}
$$

with $c>0, \delta(p):=\max \{d|1 / p-1 / 2|-1 / 2,0\}$ and $1 / p+1 / p^{\prime}=1$. The bound on the $L^{\infty}$ norm follows already from a result of Hörmander [21]. In two dimensions this simplifies to

$$
\left\|\psi_{n}\right\|_{\infty}<c E_{n}^{1 / 4} .
$$

This upper bound is sharp as is demonstrated by the eigenfunctions $Y_{l}^{m}$ for $m=0$ on the sphere $\mathcal{M}=S^{2}$. Another example is provided by the eigenfunctions of the circular billiard, where one can show that asymptotically for the rotational invariant eigenfunctions $\left\|\psi_{n}\right\|_{\infty} \sim J_{1}\left(j_{11}^{\prime}\right) E_{n}^{1 / 4}$, where $j_{11}^{\prime}$ is the first positive zero of $J_{1}^{\prime}(x)$.

The upper bound Eq. (6) has also been proven for the Dirichlet Laplacian on compact manifolds with boundary by Grieser [22]. However, for surfaces with strongly chaotic flow this upper bound is probably far away from the 
true one, and for the two-dimensional case it is conjectured [16,23] that

$$
\left\|\psi_{n}\right\|_{\infty}<c_{\varepsilon} E_{n}^{\varepsilon}, \quad \forall \varepsilon>0 .
$$

This conjecture cannot carry over to three dimensions since there are counterexamples which violate Eq. (7). Indeed, Rudnick and Sarnak showed that there exist arithmetic systems in three dimensions which possess a subsequence of Hecke eigenfunctions $\psi_{n_{j}}$ with $\left\|\psi_{n_{j}}\right\|_{\infty}>c E_{n_{j}}^{1 / 4}$ [24]. Hecke eigenfunctions are eigenfunctions of the Laplacian which are simultaneously eigenfunctions of the so-called Hecke operators which exists in the arithmetic case only. Therefore, an eigenfunction with non-degenerate eigenvalue is automatically a Hecke eigenfunction. An upper bound for Hecke eigenfunctions on certain three-dimensional arithmetic manifolds has been derived by Koyama and is given by $\left\|\psi_{n}\right\|_{\infty}<c_{\varepsilon} E_{n}^{37 / 76+\varepsilon}, \forall \varepsilon>0$ [25]. In the case of two-dimensional systems with arithmetic chaos [16,26-30] the upper bound Eq. (6) could be improved for a Hecke basis by Iwaniec and Sarnak [23]

$$
\left\|\psi_{n}\right\|_{\infty}<c_{\varepsilon} E_{n}^{5 / 24+\varepsilon}, \quad \forall \varepsilon>0 .
$$

For these systems also the lower bound

$$
\left\|\psi_{n}\right\|_{\infty} \geq c \sqrt{\ln \left(\ln E_{n}\right)}
$$

is derived.

Further results on the $L^{\infty}$ norms of eigenstates of integrable systems have been derived by Bourgain [31] and VanderKam ([32], for corrections see [33]). Bourgain claims that under certain non-recurrence conditions, which are e.g., not fulfilled for surfaces of revolution, one has $\left\|\psi_{n}\right\|_{\infty} \leq c E_{n}^{(d-1) / 4-\varepsilon}$ for some $\varepsilon>0$ which depends on the system. VanderKam showed that almost all bases of eigenfunctions on $S^{2}$ satisfy $\left\|\psi_{n}\right\|_{\infty} \leq c\left(\ln E_{n}\right)^{2}$, and that there exist bases with $\left\|\psi_{n}\right\|_{\infty} \leq c \sqrt{\ln E_{n}}$. But this extraordinary behavior is due to the high degeneracy of the eigenvalues.

For the case of quantized maps analytical and numerical results on the $L^{\infty}$ norms of Husimi densities are obtained by Nonnenmacher and Voros [34], which are analogous to the bound $\sqrt{\ln E_{n}}$ for the case of quantized flows.

Concerning the $L^{\infty}$ norms of random waves, Hejhal and Rackner [4] (see also [16]) give arguments that in analogy with the result of Salem and Zygmund [35] for random Fourier series one should have

$$
\left\|\psi_{n}\right\|_{\infty} \simeq c \sqrt{\ln E_{n}}
$$

This growth of the maximum norms of random waves can be understood by the following heuristic argument. Since the random waves vary on a scale given by the de Broglie wavelength $\lambda_{\mathrm{B}}=2 \pi / \sqrt{E}$ (see e.g., [36]), one has per dimension and per de Broglie wavelength $\lambda_{B}$ on average two extrema in a given random wave. Thus, the number of extrema $N_{\text {ext }}$ of an eigenfunction in a billiard with volume $\operatorname{vol}(D)$ is in the semiclassical limit

$$
N_{\mathrm{ext}}=2^{d} \frac{\operatorname{vol}(D)}{\lambda_{\mathrm{B}}^{d}}=\frac{E^{d / 2} \operatorname{vol}(D)}{\pi^{d}},
$$

where $d$ is the dimension of the manifold $\mathcal{M}$. The random waves, Eq. (2), have a Gaussian amplitude distribution for $N, E \rightarrow \infty$, such that $P(|f|)=(2 / \sqrt{2 \pi} \sigma) \exp \left(-|f|^{2} / 2 \sigma^{2}\right)$. The width $\sigma$ is determined by the volume of the billiard. For an amplitude $|f|$ to occur, one has to require at least $N_{\text {ext }} P(|f|)=1$. Solving this equation for $|f|$ provides an estimate of $\|f\|_{\infty}$. For $\operatorname{vol}(D)=1, d=2$ and $\sigma=1 / \sqrt{\operatorname{vol}(D)}$, one obtains

$$
\|f\|_{\infty} \simeq \sqrt{2} \sqrt{\ln E-\ln \left(2^{3 / 2} \pi^{5 / 2}\right)} \simeq \sqrt{2} \sqrt{\ln E} \text { for } E \rightarrow \infty .
$$

In this work we concentrate on the random-wave model, but we would like to mention a further model for eigenfunctions of chaotic systems which has been proposed by Zelditch [37]. He starts with a given basis of 
eigenfunctions of an auxiliary Hamilton operator on a manifold $\mathcal{M}$ and constructs a random basis by applying a randomly chosen unitary operator to this basis. These new functions are considered as a model for eigenfunctions of a chaotic Hamiltonian on the manifold. Then, he can prove for instance that almost all such bases are quantum ergodic. For the $L^{\infty}$ norms of them nothing is known. The advantage of this model is that the auxiliary basis lives on the manifold and satisfies appropriate boundary conditions, so the model eigenfunction have these properties too. This is not the case for the random-wave model Eq. (2), which can be thought of as being a local model which does not take into account the global geometry of the manifold, or boundary conditions. On the other hand, the advantage of our random waves is that they are by construction local solutions of the eigenvalue equation (1) in contrast to the random bases. Therefore, at finite energies one has a better coincidence of the local fluctuation properties, characterized by the de Broglie wavelength, of the random waves with the true eigenfunctions.

This paper is organized as follows. Section 2 concerns the distribution of the maxima of random waves, and in particular, gives a proof of an upper bound, similar to Eq. (10), including the value of the constant $c$. In Section 3, we present the numerical results for the $L^{\infty}$ norms for a number of different chaotic systems, including Euclidean billiards and arithmetic and non-arithmetic systems on surfaces of constant negative curvature, and for some randomwave ensembles, and compare them with the theoretical prediction from Section 2. We conclude with a summary of our results.

\section{The maxima of random waves}

There exist a number of results in the literature concerning the maxima of a random superposition of certain basis functions. This has started with the work of Salem and Zygmund on trigonometric series with random coefficients [35], and has been generalized by Kahane [38]. To treat the random-wave model (2) we need a generalization of this result.

Let $D \subset \mathbb{R}^{d}$ be a compact domain, and let $\mathcal{B}$ be a space of bounded real functions on $D$,

$$
\|f\|_{\infty}=\max _{\boldsymbol{x} \in D}|f(\boldsymbol{x})| \leq b
$$

for all $f \in \mathcal{B}$. Furthermore, we assume that there exists a constant $\rho>0$ such that for any finite sum $f=$ $(1 / \sqrt{N}) \sum_{n=1}^{N} a_{n} f_{n}$ with $f_{n} \in \mathcal{B}$ and $a_{n} \in \mathbb{R}$ one has

$$
\operatorname{vol}\left(A_{f}\right) \geq \frac{1}{\rho}
$$

where $A_{f}:=\left\{\boldsymbol{x} \in D \mid f(\boldsymbol{x}) \geq(1 / 2)\|f\|_{\infty}\right\}$. This means that the functions in $\mathcal{B}$ fluctuate on a length scale given by $\rho^{1 / d}$. Now we choose some probability measure $\mu\left(f_{n}\right)$ on $\mathcal{B}$ and study the random series

$$
f(\boldsymbol{x})=\frac{1}{\sqrt{N}} \sum_{n=1}^{N} a_{n} f_{n}(\boldsymbol{x}),
$$

where the $f_{n} \in \mathcal{B}$ are independently, identically distributed according to the probability measure $\mu\left(f_{n}\right)$, and the $a_{n} \in \mathbb{R}$ are chosen to be independent subnormal random variables. Recall that a random variable $a \in \mathbb{R}$ is called subnormal if the expectation value of $\mathrm{e}^{\alpha a}$ satisfies

$$
\mathbb{E}\left(\mathrm{e}^{\alpha a}\right):=\int \mathrm{e}^{\alpha a} \mathrm{~d} \sigma(a) \leq \mathrm{e}^{\alpha^{2} / 2},
$$

for $\alpha \in \mathbb{R}$, where $\sigma(a)$ denotes the probability measure of $a$. If equality holds then the random variable $a$ is normal distributed. 
Kahane has studied the distribution of $\|f\|_{\infty}$ for random series similar to Eq. (15), but with the $f_{n}$ fixed. His methods carry over with modifications to our situation leading to:

Theorem 1. Let $f$ be of the form (15) with $a_{n}$ subnormal, and assume that the bounds (13) and (14) hold, then for $\beta>(2 \rho \operatorname{vol}(D))^{-1}$

$$
\mathcal{P}\left(\|f\|_{\infty} \geq 3 b \sqrt{\ln (2 \rho \operatorname{vol}(D) \beta)}\right) \leq \frac{1}{\beta},
$$

where $\mathcal{P}(X)$ denotes the probability that the event $X$ becomes true.

Proof. We first prove the case $b=1$, the general case follows just by multiplication with $b$. We start by estimating $\mathbb{E}\left(\mathrm{e}^{\alpha f(\boldsymbol{x})}\right)$. First we consider an individual term in the series, $a_{n} f_{n}(\boldsymbol{x}) / \sqrt{N}$,

$$
\begin{aligned}
\mathbb{E}\left(\mathrm{e}^{\alpha a_{n} f_{n}(\boldsymbol{x}) / \sqrt{N}}\right)= & \iint \mathrm{e}^{\alpha a_{n} f_{n}(\boldsymbol{x}) / \sqrt{N}} \mathrm{~d} \sigma\left(a_{n}\right) \mathrm{d} \mu\left(f_{n}\right) \leq \int \mathrm{e}^{\left(\alpha f_{n}(\boldsymbol{x})\right)^{2} / 2 N} \mathrm{~d} \mu\left(f_{n}\right) \\
& \leq \int \mathrm{e}^{\alpha^{2} / 2 N} \mathrm{~d} \mu\left(f_{n}\right)=\mathrm{e}^{\alpha^{2} / 2 N},
\end{aligned}
$$

where we have used that $a_{n}$ is subnormal (16), and that $f_{n} \in \mathcal{B}$ is bounded by $b=1$. Now by the independence of $a_{n}$ and $f_{n}$ and with Eq. (18) one gets

$$
\mathbb{E}\left(\mathrm{e}^{\alpha f(\boldsymbol{x})}\right)=\mathbb{E}\left(\prod_{n=1}^{N} \mathrm{e}^{\alpha a_{n} f_{n} / \sqrt{N}}\right)=\prod_{n=1}^{N} \mathbb{E}\left(\mathrm{e}^{\alpha a_{n} f_{n} / \sqrt{N}}\right) \leq \mathrm{e}^{\alpha^{2} / 2} .
$$

According to the assumption $\operatorname{vol}\left(A_{f}\right) \geq 1 / \rho$, and with Eq. (19) we get

$$
\begin{aligned}
& \frac{1}{\rho} \mathbb{E}\left(\mathrm{e}^{\alpha\|f\|_{\infty} / 2}\right) \leq \mathbb{E}\left(\int_{A_{f}} \mathrm{e}^{\alpha\|f\|_{\infty} / 2} \mathrm{~d}^{d} x\right) \leq \mathbb{E}\left(\int_{A_{f}}\left\{\mathrm{e}^{\alpha f(\boldsymbol{x})}+\mathrm{e}^{-\alpha f(\boldsymbol{x})}\right\} \mathrm{d}^{d} x\right) \\
& \quad \leq \mathbb{E}\left(\int_{D}\left\{\mathrm{e}^{\alpha f(\boldsymbol{x})}+\mathrm{e}^{-\alpha f(\boldsymbol{x})}\right\} \mathrm{d}^{d} x\right)=\int_{D} \mathbb{E}\left(\mathrm{e}^{\alpha f(\boldsymbol{x})}+\mathrm{e}^{-\alpha f(\boldsymbol{x})}\right) \mathrm{d}^{d} x \\
& \leq 2 \operatorname{vol}(D) \mathbb{E}\left(\mathrm{e}^{\alpha f(\boldsymbol{x})}\right) \leq 2 \operatorname{vol}(D) \mathrm{e}^{\alpha^{2} / 2} .
\end{aligned}
$$

Now we estimate the probability that $\|f\|_{\infty} \geq \alpha+2 \ln (2 \operatorname{vol}(D) \beta \rho) / \alpha$ for the parameters $\alpha, \beta>0$, which will be fixed below. This is given by

$$
\mathcal{P}\left(\|f\|_{\infty} \geq \alpha+\frac{2}{\alpha} \ln (2 \operatorname{vol}(D) \beta \rho)\right)=\mathbb{E}\left(\Theta\left(\|f\|_{\infty}-\alpha-\frac{2}{\alpha} \ln (2 \operatorname{vol}(D) \beta \rho)\right)\right),
$$

where $\Theta(x)=1$ for $x \geq 0$ and $\Theta(x)=0$ for $x<0$. Since $\Theta(x) \leq \mathrm{e}^{\alpha x / 2}$ for all $\alpha>0$ we get

$$
\begin{aligned}
& \mathcal{P}\left(\|f\|_{\infty} \geq \alpha+\frac{2}{\alpha} \ln (2 \operatorname{vol}(D) \beta \rho)\right) \leq \mathbb{E}\left(\mathrm{e}^{\alpha / 2\left(\|f\|_{\infty}-\alpha-(2 / \alpha) \ln (2 \operatorname{vol}(D) \beta \rho)\right)}\right) \\
& =\mathbb{E}\left(\mathrm{e}^{\alpha\|f\|_{\infty} / 2}\right) \mathrm{e}^{-\alpha^{2} / 2} \frac{1}{2 \operatorname{vol}(D) \beta \rho} \leq \frac{1}{\beta},
\end{aligned}
$$

where the result (20) has been used in the last step.

The estimate becomes optimal if we choose $\alpha=\sqrt{\ln (2 \operatorname{vol}(D) \beta \rho)}$, and in order that the logarithm is positive we have to impose the condition $\beta>(2 \rho \operatorname{vol}(D))^{-1}$. This gives

$$
\mathcal{P}\left(\|f\|_{\infty} \geq 3 \sqrt{\ln (2 \operatorname{vol}(D) \beta \rho)}\right) \leq \frac{1}{\beta},
$$


for $\beta>(2 \rho \operatorname{vol}(D))^{-1}$, which finishes the proof of the Theorem for $b=1$. For other $b$ one applies the above proof to $f / b$, which gives Eq. (17).

If one wants to model the eigenfunctions of a chaotic system by random waves, one will choose $\mathcal{B}$ as a space of solutions of the Helmholtz equation with energy $E$, i.e., the $f_{n} \in \mathcal{B}$ satisfy $\Delta f_{n}+E f_{n}=0$, but without demanding any boundary conditions on $\partial D$. For example, plane waves as in Eq. (2) or circular waves given by Bessel functions. For such functions one expects that they fluctuate on a length of a de Broglie wavelength $\lambda_{B}=2 \pi / \sqrt{E}$, see e.g., [36]. This would imply for the parameter $\rho$ an estimate

$$
\rho \geq C^{\prime} E^{d / 2} \text {. }
$$

Applying Theorem 1 to $f=(1 / \sqrt{N}) \sum_{n=1}^{N} a_{n} f_{n}$, and choosing $\beta=E^{\delta}$ for some $\delta>0$ leads together with the assumption (24) to

$$
\mathcal{P}\left(\|f\|_{\infty} \geq 3 b \sqrt{\left(\frac{d}{2}+\delta\right) \ln (E)+\ln \left(2 \operatorname{vol}(D) C^{\prime}\right)}\right) \leq \frac{1}{E^{\delta}},
$$

for every probability measure $\mu$ on $\mathcal{B}$. Since this is valid for any $\delta>0$ we get in the limit $E \rightarrow \infty$

$$
\lim _{E \rightarrow \infty} \sup \frac{\|f\|_{\infty}}{\sqrt{\ln E}} \leq 3 b \sqrt{\frac{d}{2}},
$$

with probability one. So the maxima of the random waves do not grow faster than $\sqrt{\ln E}$. What is remarkable about this result is that Theorem 1 and therefore Eq. (26) are independent of the special properties of the functions in $\mathcal{B}$, and of the probability measure $\mu$ on $\mathcal{B}$, as long as the estimate (24) is satisfied.

In the next Section, we will compare the behavior of eigenfunctions of classically chaotic systems with the behavior of the random-wave model (2). In the setup of Theorem 1 the space $\mathcal{B}$ is now given by the set of all functions of the form $b \cos (\boldsymbol{k x}+\varepsilon)$ with $|\boldsymbol{k}|=\sqrt{E}$ and $\varepsilon \in[0,2 \pi$ [. For random waves which are normalized on the unit square with respect to the $L^{2}$ norm in the limit $N \rightarrow \infty$, one has $b=\sqrt{2}$. The space $\mathcal{B}$ is parameterized by $S^{d-1} \times[0,2 \pi]$, where $S^{d-1}$ denotes the unit sphere in $\mathbb{R}^{d}$. The measure $\mu$ on $\mathcal{B}$ is given by $(1 / 2 \pi) \mathrm{d} \nu \mathrm{d} \varepsilon$, where $\mathrm{d} \nu$ is the normalized Liouville measure on the unit-sphere. To apply Theorem 1 we need an estimate like Eq. (24) for the functions (2).

Lemma 1. For any $f$ of the form (2) with $\left|k_{n}\right|^{2}=E$ one has

$$
\operatorname{vol}\left(A_{f}\right) \geq \frac{C}{E^{d / 2}},
$$

with $C$ independent of $f$. So the parameter $\rho$ is given by $\rho=E^{d / 2} / C$

Proof. The function $f$ is of the form $f(\boldsymbol{x})=(1 / \sqrt{N}) \sum_{n=1}^{N} a_{n} \cos \left(\boldsymbol{k}_{n} \boldsymbol{x}+\varepsilon_{n}\right)$. For any unit vector $\hat{\boldsymbol{e}} \in \mathbb{R}^{n}$ one has $\left|(\hat{e} \boldsymbol{\nabla})^{l} f(\boldsymbol{x})\right| \leq E^{l / 2} C$ with $l \in \mathbb{N}$ and $C \leq \sum\left|a_{n}\right|$. Since $A_{f}=A_{c f}$ for every $c \neq 0$ we can assume that

$$
\sup \max _{x \in D}\left|\left[(\hat{e} \boldsymbol{\nabla})^{l} f\right](\boldsymbol{x})\right| E^{-l / 2}=1,
$$

where the supremum is taken over all unit vectors $\hat{e}$ and all $l \in \mathbb{N}$. Now choose $\boldsymbol{a} \in D$ such that the maximum of $|f(\boldsymbol{a})|=\|f\|_{\infty}$, and consider the Taylor series of $f(\boldsymbol{a}+s \hat{\boldsymbol{e}})$ for an arbitrary $\hat{e} \in \mathbb{R}^{d}$ with $|\hat{e}|=1$,

$$
f(\boldsymbol{a}+s \hat{\boldsymbol{e}})=\sum_{l=0}^{\infty} \frac{1}{l !}\left[(\hat{e} \boldsymbol{\nabla})^{l} f\right](\boldsymbol{a}) s^{l},
$$


which is absolutely convergent. Together with Eq. (28) one obtains

$$
|f(\boldsymbol{a}+s \hat{\boldsymbol{e}})-f(\boldsymbol{a})| \leq \sum_{l=1}^{\infty} \frac{1}{l !}\left|\left[(\hat{e} \boldsymbol{\nabla})^{l} f\right](\boldsymbol{a})\right||s|^{l} \leq \sum_{l=1}^{\infty} \frac{1}{l !}(\sqrt{E}|s|)^{l}=\mathrm{e}^{\sqrt{E}|s|}-1 .
$$

If we demand that $\mathrm{e}^{\sqrt{E}|s|}-1 \leq|f(\boldsymbol{a})| / 2$, which is equivalent to $|s| \leq \ln (1+|f(\boldsymbol{a})| / 2) / \sqrt{E}$, we have that $|f(\boldsymbol{a}+s \hat{\boldsymbol{e}})| \geq|f(\boldsymbol{a})| / 2$. So this gives the estimate

$$
\operatorname{vol}\left(A_{f}\right) \geq \operatorname{vol}\left(B\left(a, \frac{\ln (1+|f(\boldsymbol{a})| / 2)}{\sqrt{E}}\right) \cap D\right),
$$

where $B(\boldsymbol{a}, r)$ denotes the ball of radius $r$ around $\boldsymbol{a}$. The volume of the set on the right-hand side is larger than some constant times $E^{-d / 2}$ if $\partial D$ is smooth. The proof is complete if we can show that $|f(\boldsymbol{a})|=\max _{x \in D}|f(\boldsymbol{x})| \geq c>0$ for some constant $c$. If this would not be the case, then there would exist a sequence $f^{(j)}$ with $\max _{x \in D}\left|f^{(j)}(\boldsymbol{x})\right| \rightarrow 0$. But this contradicts the assumption (28) for any $j$, because for an analytic function which converges uniformly to zero, all derivatives converge to zero too.

Specializing the estimate (26) of the maxima of random waves to our case, i.e., $b=\sqrt{2}$ and $d=2$, gives

Corollory 1 ( $\mathrm{L}^{\infty}$ norms for the random-wave model). For a random function of the type (2) with $b=\sqrt{2}$ one has

$$
\lim _{E \rightarrow \infty} \sup \frac{\|f\|_{\infty}}{\sqrt{\ln E}} \leq 3 \sqrt{2} .
$$

with probability one.

\section{Numerical results}

For our numerical investigations of the $L^{\infty}$ norms we have chosen strongly chaotic systems on surfaces of constant negative curvature as well as billiards on the Euclidean plane. These systems are the same as in our studies concerning the rate of quantum ergodicity $[39,40]$.

Three systems are given by a point particle sliding freely on a surface or within a domain of constant negative Gaussian curvature providing a model for an Anosov system (see [1] and references therein). Two of them are hyperbolic triangular billiards with a domain uniquely defined by the three angles $\alpha=\pi / 2, \beta=\pi / 3$ and $\gamma=$ $\pi / 8$. On the sides $a, b$ and $c$ lying opposite to the corresponding angles the boundary conditions NND and DNN (N=Neumann, $\mathrm{D}=$ Dirichlet) are imposed leading to a non-arithmetic and an arithmetic system, respectively. The simple difference in the boundary condition leads to completely different statistics in the energy spectra [30]; whereas the rate of quantum ergodicity is, in contrast, the same for both [39]. For the 'NND' and 'DNN' billiards we have computed the first 2092 and 2099 eigenfunctions, respectively. In addition to these two triangles an asymmetric hyperbolic octagon is included in our analysis which only possesses one involution symmetry, which is always present in hyperbolic octagons. It is non-arithmetic and can easily be desymmetrized with respect to the involution symmetry. The analysis concerns the subspectrum belonging to the positive parity class comprising the first 3139 eigenfunctions.

As chaotic Euclidean billiards we choose the stadium and the cardioid billiard. The stadium billiard is proven to be a $K$-system [41]. The height of the desymmetrized billiard is chosen to be 1 , and the length of the upper horizontal line is $a=1.8$. For this system our analysis is based on computations of the first 2000 eigenfunctions for the odd-odd and for the even-even parity, i.e., Dirichlet and Neumann boundary conditions on the symmetry lines, respectively. For previous investigations of the eigenfunctions see e.g., the references in [15]. 

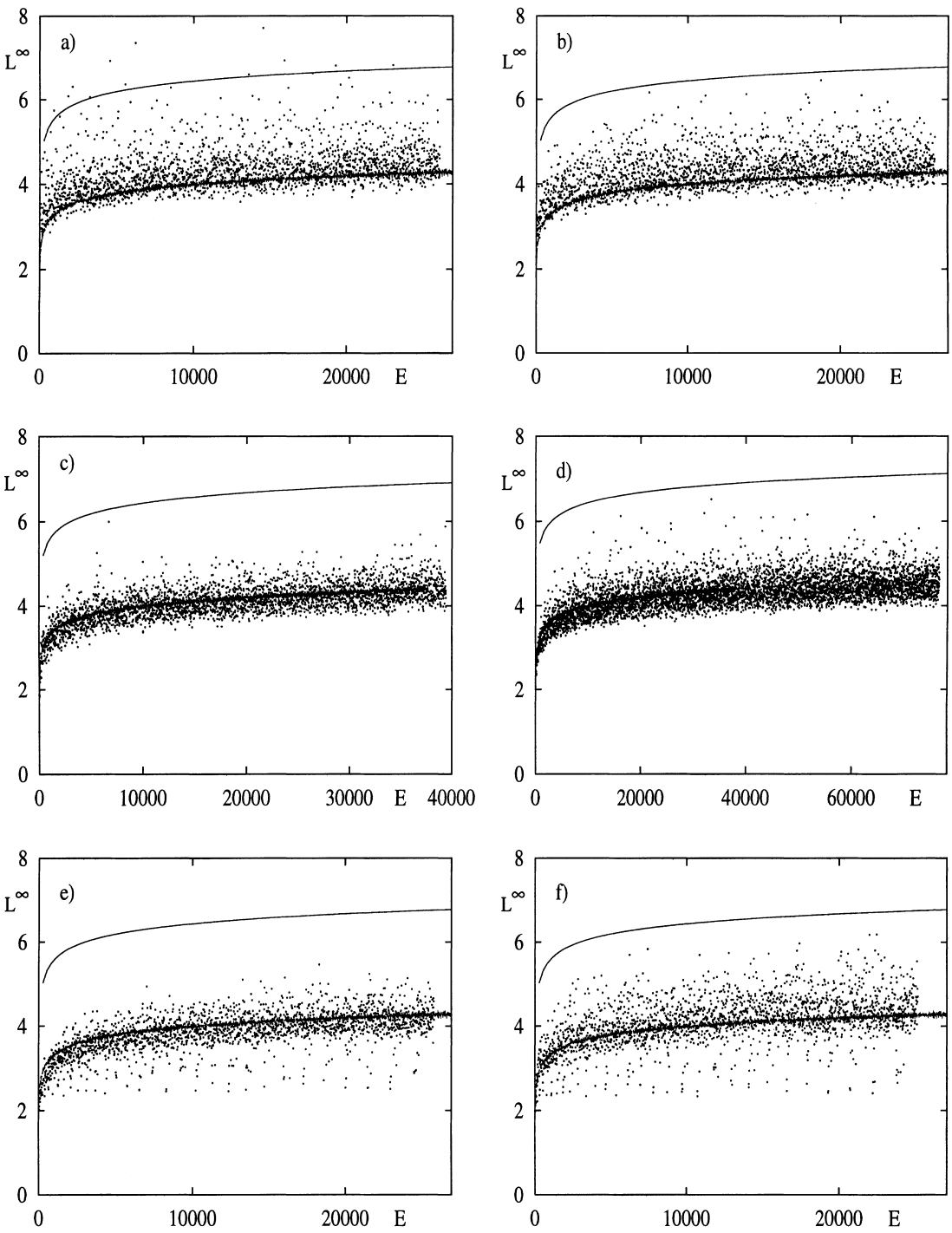

Fig. 1. The maximum norms of the hyperbolic triangular billiards with 'DNN' (a) and 'NND' (b) boundary conditions, the hyperbolic octagon (c), the cardioid billiard (d) and the stadium billiard for odd-odd (e) and for even-even (f) parity are shown. The upper curve is the function $\Omega(E)$, Eq. (33), which is two times smaller than the upper bound (32). In addition, the mean of the 200 random-wave sequences is shown for the 3000 energies $E_{n}=4 \pi n$, with $n=1, \ldots, 3000$.

The cardioid billiard is the limiting case of a family of billiards introduced in [42]. The cardioid billiard is proven to be strongly chaotic, see $[43,44]$ and references therein. The eigenvalues of the cardioid billiard have been provided by Prosen and Robnik [45] and were calculated by means of the conformal mapping technique, see e.g., [46,47]. Using these eigenvalues, our study is based on computations of the first 6000 eigenfunctions of odd symmetry, which were obtained from the eigenvalues by means of the boundary integral method $[48,49]$ using the singular value decomposition method [5], which was also used for the other systems under consideration.

To determine how well the semiclassical upper bound (32) matches the behaviour of random waves corresponding to the first few thousand eigenfunctions, we have computed 200 random-wave sequences each consisting of 3000 'eigenstates'. We generate the random waves according to Eq. (2) with $N=1000$ using random-number generators. 


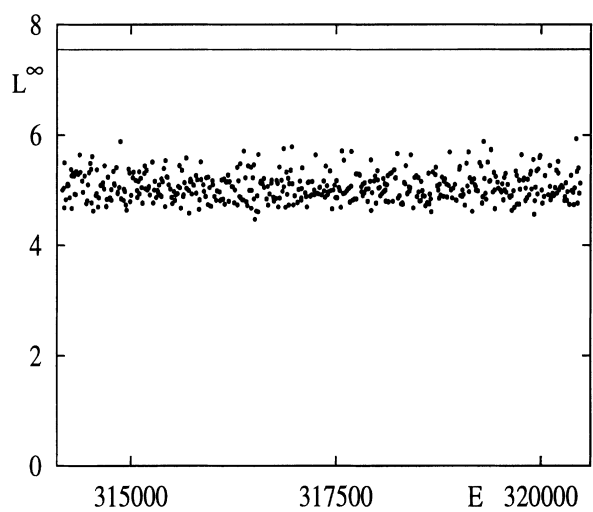

Fig. 2. The maximum norms of a very high energy range are shown for the positive parity class of the hyperbolic octagon (see also Fig. 1c) in comparison with the function $\Omega(E)$, Eq. (33). The states correspond to the 25001 th up to the 25500 th state with respect to the positive parity class.

A single random wave is normalized on the unit square with respect to the $L^{2}$ norm. The energies in a sequence are assumed to be distributed according to Weyl's law, i.e., $E_{n}=4 \pi n$, where we considered $n=1, \ldots, 3000$. For each random wave the maximum norm is computed on the unit square, and the upper bound (32) was never violated. Indeed, the upper bound (32) is also fulfilled with a coefficient $c$, which is by a factor two smaller than the value $c=3 \sqrt{2}=4.24 \ldots$ in (32) at least for our 200 numerically generated random sequences. For $c=2.0$ we found six cases in 600000 random waves, which violate the bound, whereas for $c=3 / \sqrt{2}=2.12 \ldots$ no such case is found. Therefore, in the following we will compare the maximum norms of the actual chaotic systems with the function

$$
\Omega(E)=\frac{3}{\sqrt{2}} \sqrt{\ln E}
$$

which is by a factor two smaller than the upper bound (32).

Fig. 1 shows the maximum norms belonging to the six chaotic systems. The maximum norms of the hyperbolic triangular billiard with 'DNN' and 'NND' boundary conditions are shown in Figs. 1(a,b), respectively. The norms for the hyperbolic octagon, the cardioid billiard and the stadium billiard with odd-odd and even-even parity are displayed in Figs. 1(c,d,e,f), respectively. In order to compare the norms with those of the random-wave model on the unit square, the chaotic systems have to be scaled to unit area, i.e., the energy has to be multiplied by the area of the billiard and the norm has to be multiplied by the square root of the area. Fig. 1 also shows the mean of the 200 different maximum norms of the random-wave model visible as the dark band within the cloud of dots corresponding to maximum norms of the eigenstates of the chaotic systems.

For all systems the maximum norms are in accordance with the upper bound (32). With the exception of the arithmetic system, all maximum norms are also smaller than the function $\Omega(E)$, Eq. (33). The maximum norms of the arithmetic system are larger than the maximum norms of our 200 random-wave sequences. This is likely to be caused by the underlying arithmetic structure or by the existence of Hecke operators, which relate the values of the wave functions at different locations. Thus, a difference between wave functions of arithmetic and non-arithmetic systems is found. In [39] the rate of quantum ergodicity is found to be the same for the two triangular billiards, and in [50] the amplitude distribution $P(\psi)$, the autocorrelation function $C_{\mathcal{C}}(s)$ and the distribution of the expansion coefficients with respect to hyperbolic circular waves are studied for both systems and no striking difference is observed. Only in [51] a difference in the amplitude distribution for automorphic forms is found in the case of non-compact surfaces. 


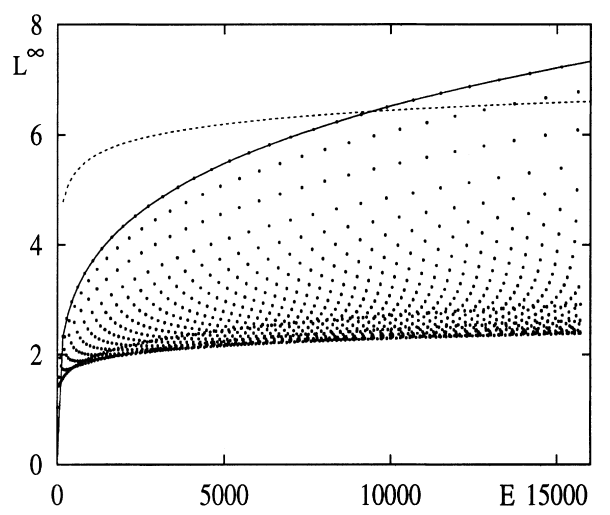

Fig. 3. The maximum norms of the first 1244 eigenfunctions of the circular billiard are shown in comparison with its asymptotic bound (full curve). The function $\Omega(E)$, Eq. (33), is shown as a dotted curve.

In Figs. 1(e,f) one observes for the stadium billiard especially low maximum norms around values slightly above two, which are due to the so-called bouncing-ball modes. These eigenfunctions localize on the bouncing-ball orbits, and can approximately be described by a product of sine functions $[14,15,52]$, such that their maximum norms are bounded. The even-even parity class shows stronger fluctuations around the mean of the random-wave sequences than the odd-odd class.

The upper bound (32) is also tested in a much higher energy range in the case of the asymmetric octagon, for which from the 25001 th state with respect to the positive parity class the next 500 eigenfunctions are computed. In Fig. 2 the maximum norms are shown in comparison with the function $\Omega(E)$, Eq. (33). It is clearly seen that in this high energy range the random-wave picture is again well supported.

To demonstrate the contrast to wave functions of integrable systems we show in Fig. 3 the maximum norms of the circular billiard in the Euclidean plane for the first 1244 eigenfunctions. For the maximum norms of the circular billiard an asymptotic upper bound can be expressed in terms of the Bessel function $J_{1}(x)$

$$
\left\|\psi_{n}\right\|_{\infty} \lesssim J_{1}\left(j_{11}^{\prime}\right) E_{n}^{1 / 4}=0.5818652 \ldots \cdot E_{n}^{1 / 4},
$$

where $j_{11}^{\prime}$ is the first positive zero of $J_{1}^{\prime}(x)$. Here, the maximum norms grow much stronger than in the case of the random-wave model or chaotic systems.

The positions at which the eigenfunctions possess their maximum norms, are shown in Fig. 4 for the six systems. In the case of the hyperbolic triangular billiards a high concentration of such positions is observed along the boundaries on which Neumann conditions are imposed. With the exception of these boundaries the locations of maximum norms look uniformly equidistributed. In the case of the hyperbolic octagon only one half of the octagon is shown because of the chosen positive parity symmetry. Here, periodic boundary conditions are imposed and, thus, no attraction of maxima along a Neumann boundary can occur. The apparent higher density of maxima towards the unit circle, which represents the boundary of the Poincare disc (see Fig. 5), is solely due to the non-Euclidean metric. In the case of the three hyperbolic systems one observes, besides effects from Neumann boundary conditions, uniformly distributed positions. For the Euclidean billiards we find for the stadium billiard with even-even parity a large number of maxima located on the boundaries with Neumann boundary conditions. In contrast to the hyperbolic systems, the positions of the maxima of the Euclidean billiards are not uniformly distributed, instead we observe some structures within the billiards. The reason for these structures is unclear and might be an effect due to the focusing parts of the boundary. These patterns have some similarity with the structure of the sums $\sum_{E_{n} \leq E}\left|\psi_{n}(\boldsymbol{x})\right|^{2}$ which are asymptotically governed by boundary effects, see [39,40]. From Bogomolny's periodic orbit analysis of 

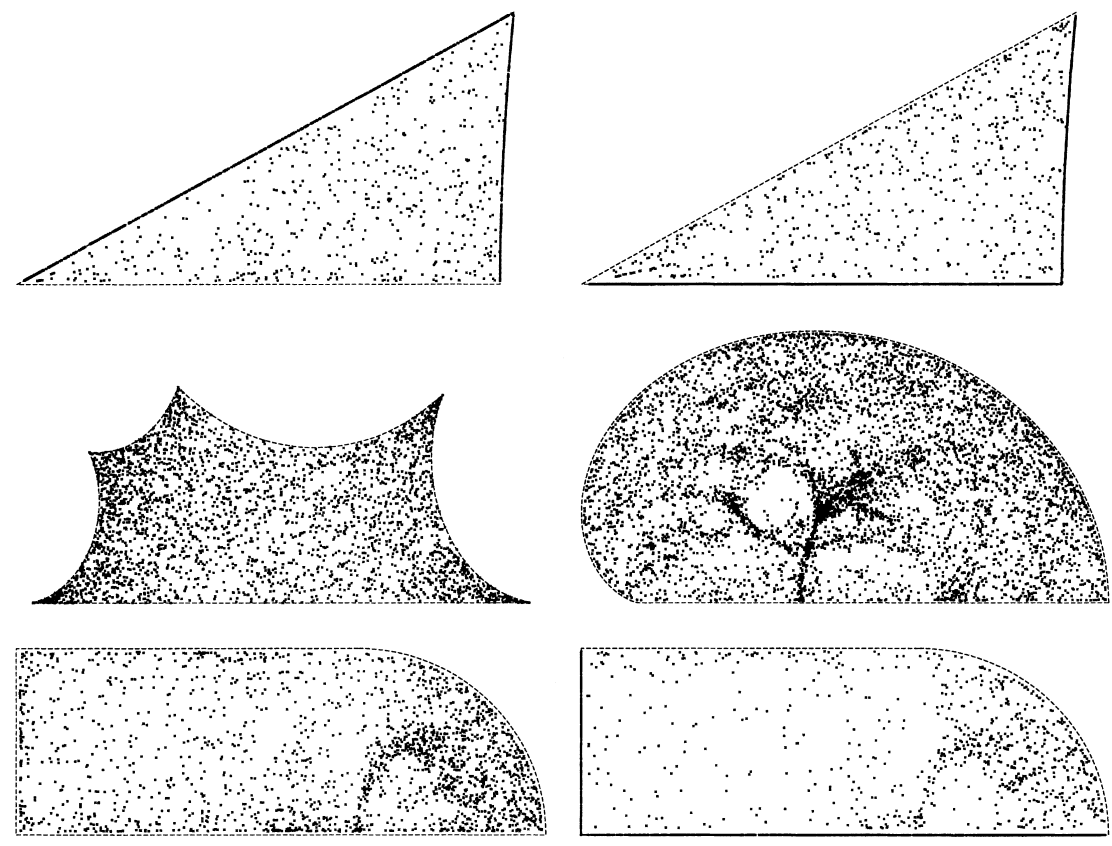

Fig. 4. The locations at which the eigenfunctions have their maximal value $\left\|\psi_{n}\right\|_{\infty}$ are displayed for the six systems as in Fig. 1.

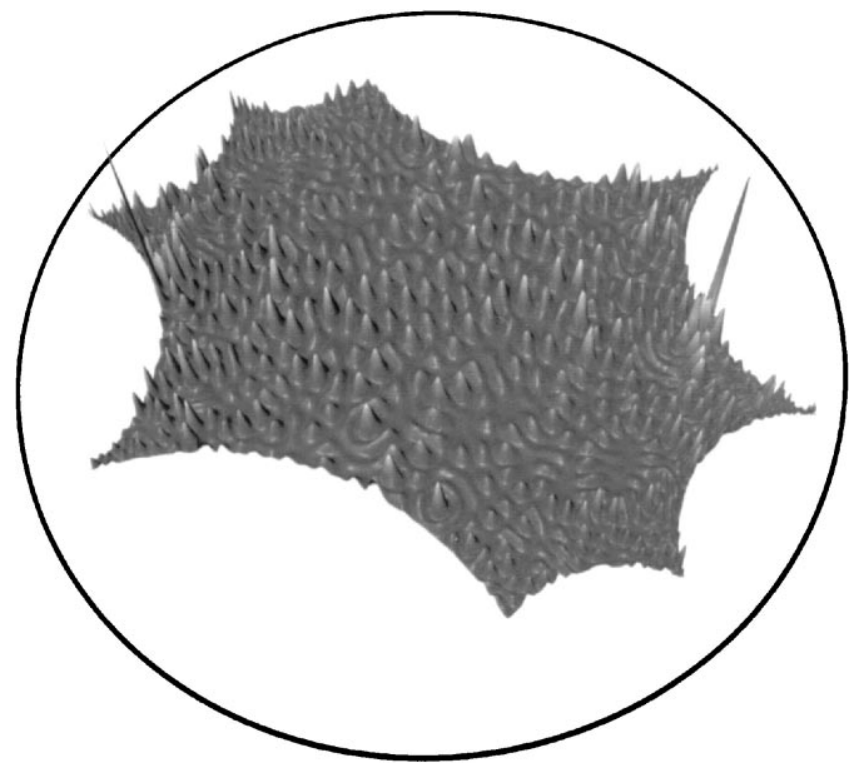

Fig. 5. The intensity $\left|\psi_{n}\right|^{2}$ for the eigenstate $n=533$. It has the largest observed maximum norm.

eigenfunctions it follows that they are expected to have maxima at focal points, or at points where periodic orbits cross [53]. Indeed, for the cardioid billiard the region with the largest accumulation of maxima corresponds to the places, where the shortest periodic orbit has its focal points.

To illustrate the fact that the maximum norm is a measure for localization effects of eigenstates we present in Figs. 5 and 6 the eigenstates with the highest maximum norms found in the hyperbolic octagon (Fig. 5) and in the cardioid 


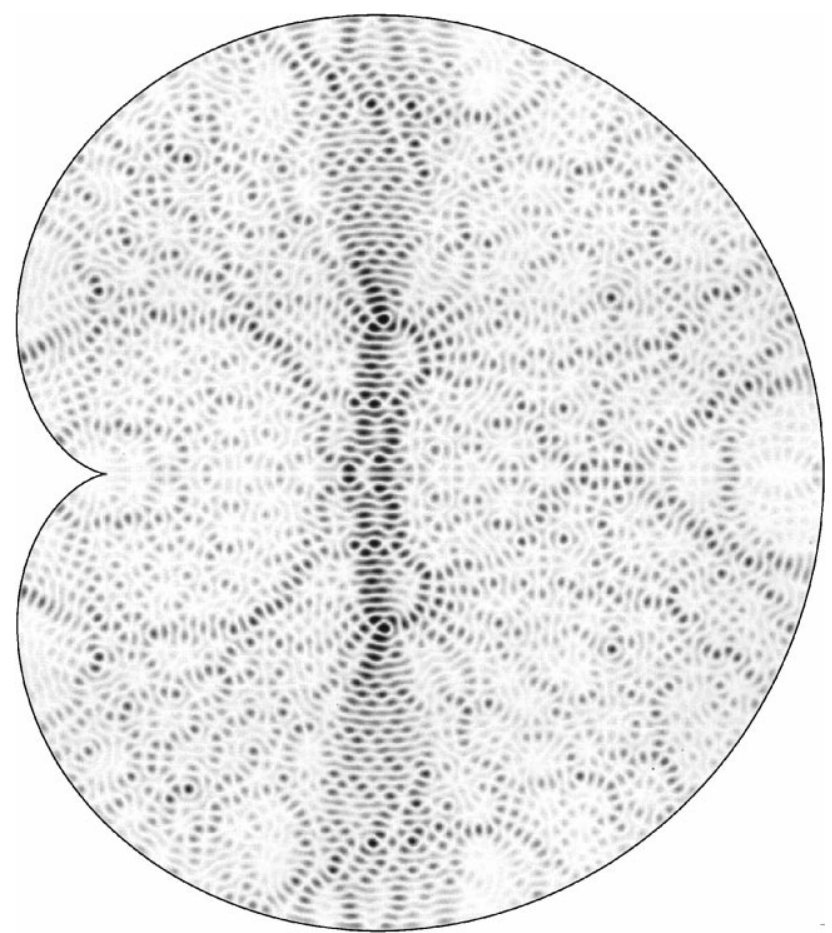

Fig. 6. Density plot of $\left|\psi_{n}\right|^{2}$ for $n=2605$ the cardioid billiard, for which the largest maximum among the first 6000 eigenfunctions occurs. The eigenfunction shows localization along the unstable period-two orbit, with the maxima located near the two focal points of the orbit.

billiard (Fig. 6). In the former case the eigenstate is strongly localized on an elliptic point of the desymmetrized group describing the octagon. Since the elliptic point lies on the boundary on which a periodic boundary condition is imposed, the peak shows up at the two corresponding boundaries. In the case of the cardioid billiard the eigenstate is strongly localized along the unstable period-two orbit. The maximum of the eigenfunction is located close to the two focal points of this orbit.

\section{Summary}

For classically strongly chaotic systems it is expected that the eigenfunctions behave like a superposition of plane waves with random amplitudes and random phases. One implication of the random-wave model is that the eigenfunctions do not show strong localization properties, which is betrayed by a very slow growth rate of the maxima of the eigenfunctions. In Section 2 an upper bound, Eq. (32), for random-wave models of eigenfunctions is derived, which is proportional to $\sqrt{\ln E}$. This bound is much smaller than the general upper bound of eigenfunctions in two-dimensional systems, which is proportional to $E^{1 / 4}$.

In Section 3 the behaviour of numerically generated random waves is compared with the upper bound Eq. (32). This comparison reveals that the right-hand side of Eq. (32) could be chosen two times smaller such that no violations of the upper bound occur. This two-fold smaller bound is compared with six strongly chaotic systems, and it is found that only the arithmetic system violates it. But the upper bound Eq. (32) is fulfilled for all six chaotic systems including the arithmetic one. Thus, a peculiar behavior is found in the eigenstates of an arithmetic system. 
Furthermore we investigate the location of the maxima of the eigenfunctions and found in the case of the Euclidean billiards some interesting patterns which seem to be related to the classical dynamics.

Our results are in accordance with the random-wave picture of eigenfunctions, and they support the existing conjectures on the behavior of the maximum norms of eigenfunctions of strongly chaotic systems.

\section{Acknowledgements}

We would like to thank Prof. F. Steiner and Dr. J. Bolte for useful discussions and Prof. M. Robnik and Dr. T. Prosen for the kind provision of the eigenvalues of the cardioid billiard. Furthermore, we thank the HLRZ at Jülich, the HLRS at Stuttgart and the Rechenzentrum of the University of Karlsruhe for the access to their computers. Fig. 5 is visualized using Geomview from The Geometry Center of the University of Minnesota and then rendered using Blue Moon Rendering Tools written by L.I. Gritz. A.B. acknowledges support by the Deutsche Forschungsgemeinschaft under contract No. DFG-Ste 241/7-2.

\section{References}

[1] M.C. Gutzwiller, Chaos in classical and quantum mechanics, Springer, Berlin, New York, 1990.

[2] M.V. Berry, Regular and irregular semiclassical wavefunctions, J. Phys. A 10 (1977) 2083-2091.

[3] S.W. McDonald, A.N. Kaufmann, Wave chaos in the stadium: statistical properties of short-wave solutions of the Helmholtz equation, Phys. Rev. A 37 (1988) 3067-3086.

[4] D.A. Hejhal, B.N. Rackner, On the topography of Mass waveforms for PSL(2, Z): experiments and heuristics, Exp. Math. 1 (1992) $275-305$.

[5] R. Aurich, F.Steiner, Statistical properties of highly excited quantum eigenstates of a strongly chaotic system, Physica D 64 (1993) $185-214$.

[6] B. Li, M. Robnik, Statistical properties of high-lying chaotic eigenstates, J. Phys. A 27 (1994) 5509-5523.

[7] A.I. Shnirelman, Ergodic properties of eigenfunctions, Usp. Math. Nauk 29 (1974) 181-182 (in Russian).

[8] A.I. Shnirelman, On the asymptotic properties of eigenfunctions in the regions of chaotic motion, in V.F. Lazutkin (Ed.), KAM Theory and Semiclassical Approximations to Eigenfunctions, Springer, Berlin, 1993

[9] S. Zelditch, Uniform distribution of eigenfunctions on compact hyperbolic surfaces, Duke. Math. J. 55 (1987) 919-941.

[10] Y. Colin de Verdière, Ergodicité et fonctions propres du laplacien, Commun. Math. Phys. 102 (1985) 111-134.

[11] P. Gérard, E. Leichtnam, Ergodic properties of eigenfunctions for the Dirichlet problem, Duke Math. J. 71 (1993) $559-607$.

[12] S. Zelditch, M. Zworski, Ergodicity of eigenfunctions for ergodic billiards, Commun. Math. Phys. 175 (1996) 673-682.

[13] E.J. Heller, Bound-state eigenfunctions of classically chaotic Hamiltonian systems: scars of periodic orbits, Phys. Rev. Lett. 53 (1984) $1515-1518$

[14] G. Tanner, How chaotic is the stadium billiard? a semiclassical analysis, J. Phys. A 30 (1997) 2863-2888.

[15] A. Bäcker, R. Schubert, P. Stifter, On the number of bouncing-ball modes in billiards, J. Phys. A 30 (1997) 6783-6795.

[16] P. Sarnak, Arithmetic quantum chaos, Israel Math. Conf. Proc. 8 (1995) 183-236.

[17] W. Luo, P. Sarnak, Quantum ergodicity of eigenfunctions on $\mathrm{PSL}_{2}(\mathbb{Z}) \backslash H^{2}$, Publ. Math. Inst. Hautes Etud. Sci. 81 (1995) $207-237$.

[18] R. Aurich, F. Steiner, Quantum eigenstates of a strongly chaotic system and the scar phenomenon, Chaos Solitons Fractals 5 (1995) $229-255$.

[19] O.M. Auslaender, S. Fishman, Exact eigenfunctions of a chaotic system, Preprint, chao-dyn/9707021, 1997.

[20] A. Seeger, C.D. Sogge, Bounds for eigenfunctions of differential operators, Indiana Univ. Math. J. 38 (1989) 669-682.

[21] L. Hörmander, The spectral function for an elliptic operator, Acta Math. 127 (1968) 193-218.

[22] D. Grieser, Uniform bounds for eigenfunctions of the Laplacian on manifolds with boundary, Preprint, 1997.

[23] H. Iwaniec, P. Sarnak, $L^{\infty}$ norms of eigenfunctions of arithmetic surfaces, Ann. Math. 141 (1995) 301-320.

[24] Z. Rudnick, P. Sarnak, The behaviour of eigenstates of arithmetic hyperbolic manifolds, Commun. Math. Phys. 161 (1994) $195-213$.

[25] S.Y. Koyama, $L^{\infty}$-norms of eigenfunctions for arithmetic hyperbolic three-manifolds, Duke Math. J. 77 (1995) $799-817$.

[26] R. Aurich, F. Steiner, On the periodic orbits of a strongly chaotic system, Physica D 32 (1988) 451-460.

[27] E.B. Bogomolny, B. Georgeot, M.-J. Giannoni, C. Schmit, Chaotic billiards generated by arithmetic groups, Phys. Rev. Lett. 69 (1992) $1477-1480$.

[28] J. Bolte, G. Steil, F. Steiner, Arithmetical chaos and violation of universality in energy level statistics, Phys. Rev. Lett. 69 (1992) $2188-2191$.

[29] J. Bolte, Some studies on arithmetical chaos in classical and quantum mechanics, Int. J. Mod. Phys B 7 (1993) 4451-4553.

[30] R. Aurich, F. Scheffler, F. Steiner, On the subtleties of arithmetical quantum chaos, Phys. Rev. E 51 (1995) 4173-4189.

[31] J. Bourgain, Eigenfunction bounds for compact manifolds with integrable geodesic flow, Preprint, IHES/M/93/49, 1993.

[32] J.M. VanderKam, $L^{\infty}$ norms and quantum ergodicity on the sphere, Int. Math. Res. Notices 7 (1997) 329-347. 
[33] J.M. VanderKam, Int. Math. Res. Notices 1 (1998) 65.

[34] S. Nonnenmacher, A. Voros, Chaotic eigenfunctions in phase space, Preprint, chao-dyn/9711016, 1997.

[35] R. Salem, A. Zygmund, Some properties of trigonometric series whose terms have random signs, Acta Math. 91 (1954) $245-301$.

[36] H. Donnelly, C. Fefferman, Growth and geometry of eigenfunctions of the Laplacian, in Analysis and partial differential equations: a collection of papers dedicated to Mischa Cotlar, Lecture Notes Pure Appl. Math. 122 (1990) 635-655.

[37] S. Zelditch, A random matrix model for quantum mixing, Int. Math. Res. Notices 3 (1996) 115-137.

[38] J.-P. Kahane, Some Random Series of Functions, 2nd edn., Cambridge University Press, Cambridge, 1985.

[39] R. Aurich, M. Taglieber, On the rate of quantum ergodicity on hyperbolic surfaces and billiards, Physica D 118 (1998) 84-102.

[40] A. Bäcker, R. Schubert, P. Stifter, Rate of quantum ergodicity in Euclidean billiards, Phys. Rev. E 57 (1998) 5425-5447.

[41] L.A. Bunimovich, On the ergodic properties of nowhere dispersing billiards, Commun. Math. Phys. 65 (1979) 295-312.

[42] M. Robnik, Classical dynamics of a family of billiards with analytic boundaries, J. Phys. A 16 (1983) 3971-3986.

[43] C. Liverani, M.P. Wojtkowski, Ergodicity in Hamiltonian systems, in: C.K.R.T. Jones, U. Kirchgraber, H.O. Walther (Eds.), Dynamics Reported. Expositions in Dynamical Systems. New series. Vol. 4, Springer, Berlin, 1995, pp. 130-202.

[44] N.I. Chernov, C.Haskell, Nonuniformly hyperbolic $K$-systems are Bernoulli, Ergod. Th. Dynam. Sys. 16 (1996) $19-44$.

[45] T. Prosen, M. Robnik, private communication.

[46] M. Robnik, Quantising a generic family of billiards with analytic boundaries, J. Phys. A 17 (1984) 1049-1074.

[47] T. Prosen, M. Robnik, Energy level statistics in the transition region between integrability and chaos, J. Phys. A 26 (1993) $2371-2387$.

[48] R.J. Riddel Jr., Boundary-distribution solution of the Helmholtz equation for a region with corners, J. Comp. Phys. 31 (1979) $21-41$.

[49] M.V. Berry, M. Wilkinson, Diabolical points in the spectra of triangles, Proc. R. Soc. London Ser. A 392 (1984) 15-43.

[50] M. Taglieber, Quantenergodizität in zwei hyperbolischen Dreiecks-Billards, Diploma Thesis, Universität Ulm, 1997.

[51] D.A. Hejhal, On value distribution properties of automorphic functions along closed horocycles, in: I. Laine et al. (Eds.), 16th Rolf Nevanlinna colloquium, Proc. Int. Conference held in Joensuu, Finland, 1-5 August 1995, Walter de Gruyter, Berlin, 1996, pp. $39-52$.

[52] Y.Y. Bai, G. Hose, K. Stefański, H.S. Taylor, Born-Oppenheimer adiabatic mechanism for regularity of states in the quantum stadium billiard, Phys. Rev. A 31 (1985) 2821-2826.

[53] E.B. Bogomolny, Smoothed wave functions of chaotic quantum systems, Physica D 31 (1988) 169-189. 